\title{
Entrenamiento cognitivo combinado con ejercicios aeróbicos en pacientes con esclerosis múltiple: estudio piloto
}

\author{
Rodneys M. Jiménez-Morales, Luis F. Herrera-Jiménez, Yanet Macías-Delgado, Yunia T. Pérez-Medinilla, \\ Sara M. Díaz-Díaz, Cristina Forn
}

Introducción. Las evidencias científicas asociadas a la efectividad de distintas técnicas de rehabilitación cognitiva todavía
resultan contradictorias.

Objetivo. Comparar un programa de entrenamiento combinado (físico y cognitivo) frente a un programa de entrenamiento físico y observar su eficacia sobre la optimización de las funciones cognitivas en pacientes con esclerosis múltiple (EM).

Pacientes y métodos. Se realizó un estudio experimental en 32 pacientes con EM. Los pacientes se distribuyeron en dos grupos: 16 al grupo experimental (entrenamiento cognitivo combinado con ejercicios aeróbicos) y 16 al grupo control (ejercicios aeróbicos). La intervención se planificó para seis semanas combinando tareas cognitivas mediante un juego de tablero dinámico de cubos y signos (TaDiCS ${ }^{\circledR}$ ) y un programa de ejercicios aeróbicos. Se aplicó la batería breve repetible de tests neuropsicológicos y el test de Stroop para evaluar el rendimiento cognitivo. Además, se administró el inventario de depresión de Beck.

Resultados. Se encontraron diferencias significativas en el análisis intergrupo después de la intervención en las variables aprendizaje y memoria a largo plazo visuoespacial $(p=0,000)$, atención $(p=0,026)$ y control inhibitorio $(p=0,007)$. Asimismo, en el análisis intragrupo se encontraron diferencias significativas en estas variables y en la velocidad en el procesamiento de la información en el grupo que recibió el entrenamiento combinado. Estos pacientes también mostraron una mejoría significativa en el estado de ánimo $(p=0,043)$.

Conclusión. El entrenamiento cognitivo combinado con los ejercicios aeróbicos resulta eficaz para mejorar el funcionamiento cognitivo.

Palabras clave. Aprendizaje y memoria. Atención. Ejercicios aeróbicos. Entrenamiento cognitivo. Esclerosis múltiple. Funciones cognitivas. Velocidad en el procesamiento de la información.

\section{Introducción}

La esclerosis múltiple (EM) es una enfermedad autoinmune, desmielinizante y degenerativa del sistema nervioso central con manifestaciones clínicas heterogéneas, entre las que destacan las alteraciones sensitivas y motoras, además del deterioro cognitivo. Las alteraciones cognitivas afectan un 43-65\% de los pacientes valorados en los servicios de atención sociosanitaria [1,2], y producen un gran impacto en la vida diaria de estos pacientes en el ámbito personal, laboral y social $[3,4]$. El perfil de alteración cognitiva de la EM incluye déficits en la velocidad del procesamiento de la información, y alteraciones de las funciones atencionales, mnésicas y ejecutivas [5-7].

Varias estrategias de rehabilitación cognitiva se han empleado para reducir el impacto cognitivo en los pacientes con EM. Estas estrategias han incluido intervenciones específicas sobre la atención, aprendizaje y memoria, y funciones ejecutivas [8-12], además de emplear otras intervenciones multimodales en las que se combinaban las terapias cognitivas y las psicoterapéuticas [13].

A pesar de que se han realizado distintos estudios destinados a valorar la eficacia de la rehabilitación cognitiva en los pacientes con EM, una reciente revisión sobre el tema resalta evidencias contradictorias sobre la efectividad de las distintas técnicas de rehabilitación cognitiva [14].

Por otra parte, la asociación entre el rendimiento físico y la mejora cognitiva se ha descrito en poblaciones clínicas como la demencia $[15,16]$ y, de forma más reciente, la EM [17]. A este respecto, se han realizado hasta el momento dos estudios en los que se describe que los pacientes con EM, después de realizar un proceso de rehabilitación física, mejoran su rendimiento cognitivo $[18,19]$ y otros síntomas asociados a la enfermedad, como el estado emocional o la fatiga [18].

Aunque parece evidente que un entrenamiento físico puede producir una mejora directa sobre las funciones cognitivas, hasta ahora pocos trabajos han estudiado la eficacia de programas de entrena-
Departamento de Psicología Básica, Clínica y Psicobiología; Facultad de Ciencias Humanas y Sociales; Universitat Jaume I; Castellón, España (C. Forn). Departamento de Psicología; Universidad Central Marta Abreu de las Villas; Santa Clara, Cuba (L.F. Herrera-Jiménez). Departamento de MatemáticaFísica; Universidad de Sancti Spiritus José Martí Pérez; Sancti Spíritus, Cuba (Y.T. Pérez-Medinilla). Departamento de Psicología (R.M. Jiménez-Morales, Y. Macías-Delgado); Departamento de Medicina Física y Rehabilitación (S.M. Díaz-Díaz); Hospital de Rehabilitación Doctor Faustino Pérez Hernández; Sancti Spíritus, Cuba.

Correspondencia:

Dr. Rodneys M. Jiménez Morales. Departamento de Psicología. Hospital de Rehabilitación Doctor Faustino Pérez Hernández. Ctra. Zaza, km 1,5. CP 60100. Santi Spíritus, Cuba.

E-mail:

rjimenez.ss@infomed.sld.cu

Aceptado tras revisión externa: 19.01.17.

Cómo citar este artículo: Jiménez-Morales RM, HerreraJiménez LF, Macías-Delgado Y, Pérez-Medinilla YT, Díaz-Díaz SM, Forn C. Entrenamiento cognitivo combinado con ejercicios aeróbicos en pacientes con esclerosis múltiple: estudio piloto. Rev Neurol 2017; 64: 489-95.

(c) 2017 Revista de Neurología 
Figura. Diagrama de flujo que ilustra la participación de los pacientes en el proceso de investigación.

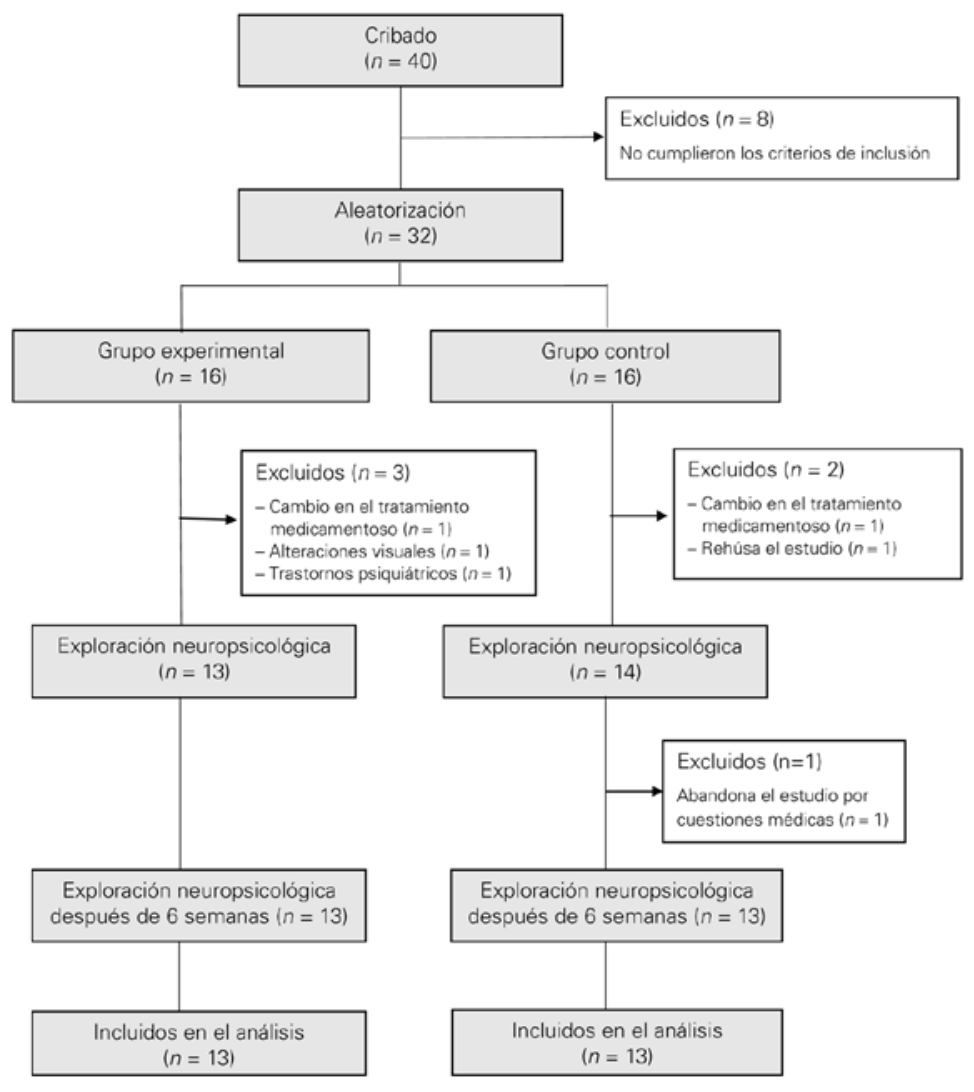

miento físico o entrenamiento combinado (físico y cognitivo) sobre el rendimiento cognitivo en pacientes con EM. Atendiendo a la escasa bibliografía existente al respecto, el principal objetivo del presente estudio fue comparar un programa de entrenamiento combinado (físico y cognitivo) frente a un programa de entrenamiento físico y observar su eficacia sobre la optimización de las funciones cognitivas en un grupo de pacientes con EM.

\section{Pacientes y métodos}

\section{Muestra}

Se seleccionaron para el estudio 32 pacientes diagnosticados de EM remitente recurrente, siguiendo los criterios de McDonald et al [20], valorados y tratados en el Hospital Provincial de Rehabilitación Dr. Faustino Pérez Hernández de Sancti Spíritus, Cuba. Los criterios de inclusión fueron:
- Pacientes $>18$ años y $<65$ años.

- Duración de la enfermedad $\leq 20$ años.

- No encontrarse durante el período de evaluación e intervención en período de recidiva (seis meses tras el último brote).

- Voluntariedad del paciente y previa información de acuerdo con la declaración de Helsinki.

Todos los pacientes fueron valorados con la escala neurológica Expanded Disability Status Scale.

Los pacientes se distribuyeron aleatoriamente en un grupo experimental (GE; $n=13$ ) y un grupo control (GC; $n=13)$. Las razones de exclusión de algunos pacientes en el proceso de investigación hasta completar el entrenamiento se describen en la figura.

Los datos clínicos y demográficos para ambos grupos se presentan en la tabla I. No se observan diferencias significativas en las distintas variables demográficas ni clínicas entre el GE y el GC.

Los pacientes seleccionados para el estudio fueron informados sobre la importancia de la investigación y se registró su consentimiento de participación. El proyecto fue aprobado por el comité de ética de la investigación y el comité científico del Hospital Provincial de Rehabilitación, en Sancti Spíritus, Cuba.

\section{Evaluación neuropsicológica}

Todos los participantes fueron evaluados con la batería breve repetible de tests neuropsicológicos de Rao, traducida y baremada a la población de habla castellana por Sepulcre et al [21]. Según Rao et al [22], esta batería cuenta con unos índices de sensibilidad del $71 \%$ y de especificidad del $94 \%$ para valorar el deterioro cognitivo en pacientes con EM.

Esta batería está formada por tests que evalúan las áreas cognitivas más afectadas en la EM y que son los siguientes [22]:

- Prueba de memoria selectiva (Selective Reminding Test, SRT), que evalúa el aprendizaje y la memoria verbal a corto y largo plazo.

- Prueba de memoria espacial (Spatial Recall Test, SPART 10/36), que valora el aprendizaje y la memoria a largo plazo visuoespacial.

- Prueba de modalidades de dígitos y símbolos (Symbol Digit Modalities Test, SDMT), que valora la atención.

- Prueba de adición auditora consecutiva (Paced Auditory Serial Addition Test, PASAT), que evalúa la velocidad del procesamiento de la información.

- Generación de lista de palabras (Word List Generation, WLG), que evalúa la fluidez fonética verbal. 
Se aplicó el test de Stroop interferencia palabra-color, que valora la capacidad inhibitoria, y el inventario de depresión de Beck (IDB) con el objetivo de valorar el estado de ánimo. La administración del IDB está admitida y recomendada por el Goldman Consensus Group para valorar el estado de ánimo en pacientes con EM, aunque se recomienda para su interpretación clínica un punto de corte de 13 puntos debido a la inclusión en el cuestionario de ítems que incluyen síntomas vegetativos que son característicos de la EM y podrían dar lugar a falsos diagnósticos [23].

\section{Programa de rehabilitación aplicado}

Los pacientes que formaron parte del GE realizaron un entrenamiento combinado de actividades cognitivas y un programa de ejercicios aeróbicos durante seis semanas, con una frecuencia de cinco veces por semana. Los ejercicios aeróbicos de resistencia graduada se distribuyeron en dos sesiones de mañana y tarde. En la sesión de mañana realizaban un ejercicio de tapiz rodante WNQ-7000a que alcanzó gradualmente un tiempo máximo de 30 minutos, mientras que en la sesión de tarde realizaban bicicleta ergométrica en un intervalo de tres a $10 \mathrm{minu-}$ tos de forma creciente. Ambos son equipos de mecanoterapia que se emplearon para el entrenamiento de la capacidad cardiorrespiratoria.

Para el programa de entrenamiento cognitivo se desarrolló un juego de tablero (TaDiCS ${ }^{\circledR}$ ) que tuvo en cuenta un conjunto de tareas cognitivas con diferentes niveles de complejidad sobre la base del modelo clínico de la atención de Sohlberg y Mateer [24], así como el modelo de solución de problemas postulado por Luria [25]. Las tareas seleccionadas fueron: rastreo visual y velocidad visuomotora (modalidad visual), atención sostenida y selectiva (modalidad auditiva y visual), atención alternante (modalidad auditiva y visual) y solución de problemas práctico-constructiva. El entrenamiento cognitivo tuvo una duración de 45 minutos diarios para un total de cuatro horas semanales. Las actividades siempre se realizaron después de los ejercicios físicos.

Los pacientes que participaron en el GC conservaron las características del programa de ejercicios aeróbicos similares a las del GE (duración, frecuencia e intensidad), pero sin la aplicación del entrenamiento cognitivo.

\section{Procedimiento}

Se efectuó una sesión educativa de una hora durante la primera semana de tratamiento con el objetivo
Tabla I. Características clínicas y demográficas del grupo de pacientes sometidos a entrenamiento combinado (GE) y el grupo control (GC).

\begin{tabular}{lcccc}
\hline & GE $(n=13)$ & GC $(n=13)$ & $t$ & $p$ \\
\hline Edad (años) & $44,62 \pm 9,70$ & $43,23 \pm 13,52$ & 0,300 & 0,879 \\
\hline Nivel educativo (años) & $15,46 \pm 4,09$ & $15,15 \pm 3,89$ & 0,196 & 0,846 \\
\hline \multicolumn{1}{c}{ Femenino } & $9(69,2 \%)$ & $10(76,9 \%)$ & - & - \\
\cline { 2 - 5 } Sexo & $4(30,7 \%)$ & $3(23,1 \%)$ & - & - \\
\hline $\begin{array}{l}\text { Masculino } \\
\text { Expanded Disability Status Scale }\end{array}$ & $4,70 \pm 1,37$ & $4,19 \pm 925$ & 1,169 & 0,254 \\
\hline $\begin{array}{l}\text { Tiempo medio de duración } \\
\text { de la enfermedad (años) }\end{array}$ & $10,38 \pm 4,07$ & $8,54 \pm 6,27$ & 0,891 & 0,382 \\
\hline
\end{tabular}

de explicar a todos los participantes los beneficios de los entrenamientos en el funcionamiento cerebral y la discapacidad física de los pacientes con EM.

Ambos grupos de estudio (GE y GC) fueron evaluados pre y postratamiento con la misma batería, pero con distintas versiones (versión A y B), para controlar un posible efecto aprendizaje. La valoración postratamiento se realizó al finalizar la sexta semana.

\section{Análisis estadísticos}

Los análisis estadísticos se efectuaron con el programa SPSS v. 15. Se realizaron pruebas $t$ para comparar ambos grupos de estudio (GE y GC) en cuanto a las variables demográficas y clínicas de interés.

La eficacia del entrenamiento combinado compuesto por tareas de rehabilitación cognitiva y ejercicios aeróbicos se comprobó mediante un análisis ANOVA intergrupo, en el que se incluyeron las comparaciones de los tests entre los grupos pre y postratamiento.

Para evaluar otros cambios en el funcionamiento cognitivo generados por el efecto del programa de entrenamiento combinado, se aplicó un ANOVA intragrupo pre y postratamiento en ambos grupos. Se consideraron las diferencias significativas en todos los casos cuando $p<0,05$.

\section{Resultados}

El análisis intergrupo evidenció cómo los pacientes con EM del GE que participaron en el programa de entrenamiento intensivo que combinó el juego de tablero (TaDiCS) y los ejercicios aeróbicos mejora- 
Tabla II. Análisis intergrupo de las medidas neuropsicológicas en el grupo experimental y el grupo control.

\begin{tabular}{|c|c|c|c|c|c|c|c|c|}
\hline & Grupo experimental & Grupo control & \multirow{2}{*}{$F$} & & Grupo experimental & Grupo control & \multirow{2}{*}{$F$} & \multirow{2}{*}{$p$} \\
\hline & \multicolumn{2}{|c|}{ Antes } & & & \multicolumn{2}{|c|}{ Después } & & \\
\hline SRT-R & $25,00 \pm 8,30$ & $29,00 \pm 9,24$ & 1,35 & 0,257 & $26,31 \pm 7,38$ & $31,31 \pm 6,47$ & 3,37 & 0,079 \\
\hline SPART 10/36 & $13,46 \pm 4,54$ & $15,23 \pm 5,26$ & 0,84 & 0,368 & $20,08 \pm 4,54$ & $17,62 \pm 3,12$ & 2,59 & 0,120 \\
\hline SPART 10/36 D & $6,38 \pm 1,19$ & $5,46 \pm 1,27$ & 3,66 & 0,068 & $7,46 \pm 1,05$ & $5,31 \pm 1,38$ & 20,10 & $0,000^{a}$ \\
\hline SDMT & $29,62 \pm 9,55$ & $31,23 \pm 5,07$ & 0,29 & 0,595 & $38,23 \pm 7,99$ & $32,00 \pm 5,13$ & 5,59 & $0,026^{b}$ \\
\hline WLG fonético & $19,38 \pm 7,62$ & $17,08 \pm 7,10$ & 0,64 & 0,432 & $24,23 \pm 8,74$ & $19,15 \pm 5,97$ & 2,99 & 0,097 \\
\hline WLG semántico & $23,00 \pm 6,68$ & $22,31 \pm 7,40$ & 0,06 & 0,804 & $28,00 \pm 6,03$ & $25,31 \pm 5,75$ & 1,36 & 0,355 \\
\hline $\begin{array}{l}\text { Stroop interferencia } \\
\text { palabra-color }\end{array}$ & $27,23 \pm 9,05$ & $31,08 \pm 6,42$ & 1,56 & 0,223 & $38,38 \pm 3,43$ & $33,00 \pm 5,66$ & 8,61 & $0,007^{a}$ \\
\hline IDB & $15,08 \pm 7,89$ & $14,00 \pm 6,86$ & 0,14 & 0,714 & $9,77 \pm 4,21$ & $9,08 \pm 2,14$ & 0,28 & 0,602 \\
\hline
\end{tabular}

IDB: inventario de depresión de Beck; PASAT: Paced Auditory Serial Addition Task; SDMT: Symbol Digit Modalities Test; SPART 10/36: Spatial Recall Test 10/36; SPART 10/36 D: Spatial Recall Test 10/36 Delayed; SRT-D: Selective Reminding Test Delayed Recall; SRT-R: Selective Reminding Test Long-Term Retrieval; SRT-S: Selective Reminding Test Long-Term Storage; WLG: Word List Generation. a $p \leq 0,01 ;{ }^{b} p \leq 0,05$ entre grupos.

ron significativamente al compararse con el GC en el rendimiento de pruebas de atención (SDMT; $p=$ 0,026), memoria a largo plazo visuoespacial (SPART $10 / 36 \mathrm{D} ; p=0,000$ ) y control inhibitorio (Stroop interferencia palabra-color; $p=0,007$ ) (Tabla II).

Del mismo modo, los resultados de la comparación intragrupo mostraron cambios significativos en el GE en los test relacionados con la velocidad del procesamiento de la información (PASAT 3; $p=$ 0,044 ), atención (SDMT; $p=0,02$ ), aprendizaje y memoria visuoespacial a corto (SPART 10/36; $p=$ $0,001$ ) y a largo plazo (SPART SRT-D; $p=0,022)$, así como capacidad de inhibición de respuesta (Stroop; $p=0,000)$. Estos pacientes también mostraron una mejoría significativa en el estado de ánimo (IDB; $p=0,043)$. El análisis intragrupo también demostró cambios significativos en el GC en medidas de velocidad de procesamiento de la información (PASAT 3; $p=0,031) y$, al igual que en el GE, estos pacientes mejoraron su estado de ánimo (IDB; $p=$ 0,021) (Tabla III).

\section{Discusión}

El objetivo era comparar un programa de entrenamiento combinado (físico y cognitivo) frente a un programa de entrenamiento físico y observar su eficacia sobre la optimización de las funciones cognitivas en un grupo de pacientes con EM remitente recurrente. Teniendo en cuenta los resultados descritos en la bibliografía sobre la eficacia de la rehabilitación combinada (cognitiva y física), se mantuvo la hipótesis de que el grupo que realizó el entrenamiento combinado presentaría un mejor rendimiento cognitivo general que el GC, que se entrenó únicamente en la modalidad de ejercicios aeróbicos.

Como se esperaba, los resultados muestran que el GE presentó un mejor rendimiento cognitivo respecto al GC. De forma más específica, los pacientes del GE mostraron una mejoría significativa en las tareas de atención, aprendizaje y memoria a largo plazo visuoespacial, así como en funciones ejecutivas como la capacidad de inhibición. Por otra parte, 
Tabla III. Análisis intragrupo de las medidas neuropsicológicas en el grupo experimental y el grupo control.

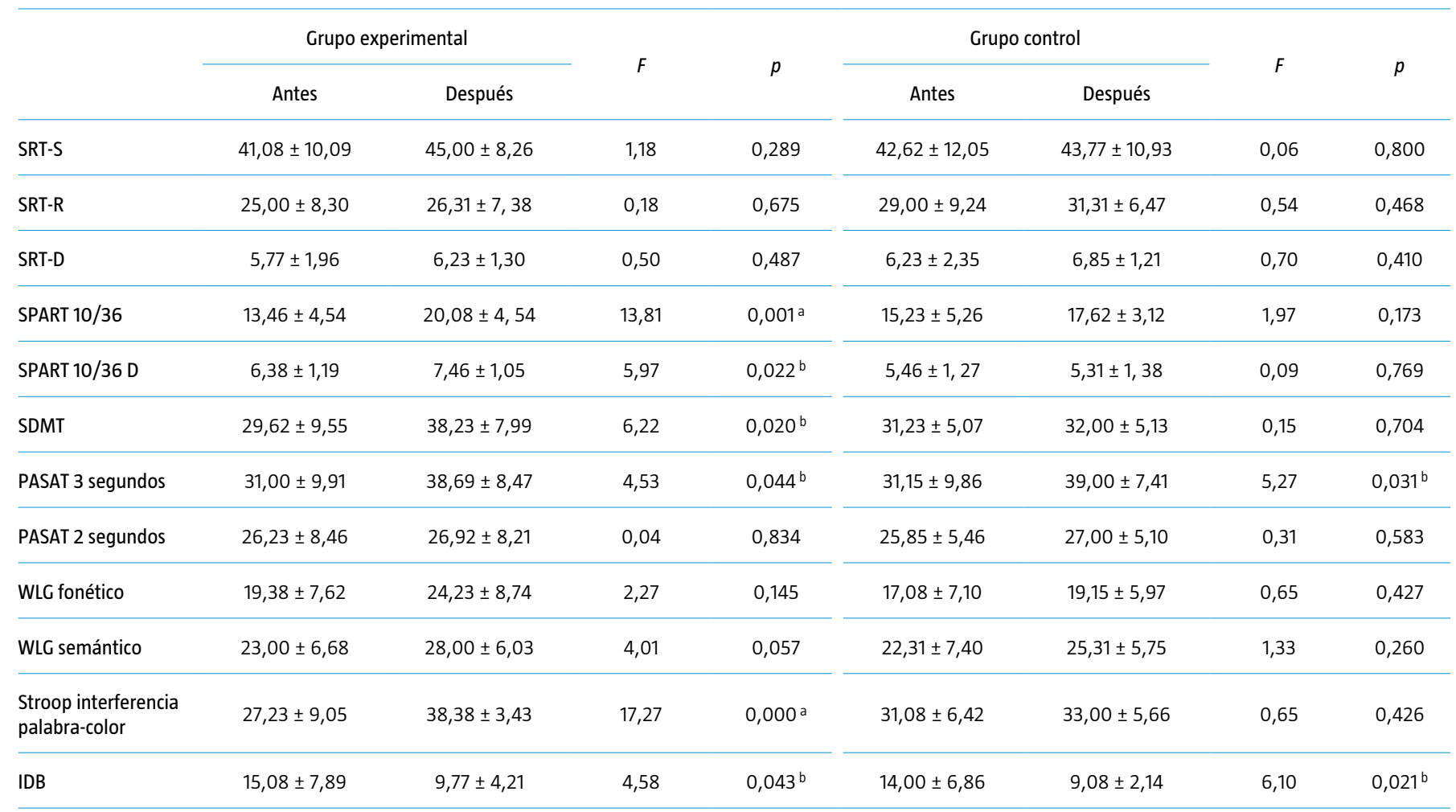

IDB: inventario de depresión de Beck; PASAT: Paced Auditory Serial Addition Task; SDMT: Symbol Digit Modalities Test; SPART 10/36: Spatial Recall Test 10/36; SPART 10/36 D: Spatial Recall Test 10/36 Delayed; SRT-D: Selective Reminding Test Delayed Recall; SRT-R: Selective Reminding Test Long-Term Retrieval; SRT-S: Selective Reminding Test Long-Term Storage; WLG: Word List Generation. ${ }^{\mathrm{a}} \mathrm{p} \leq 0,01 ;{ }^{\mathrm{b}} p \leq 0,05$ en cada grupo.

el análisis intragrupo del GE evidenció una vez más que, después del programa de intervención combinada, los pacientes mejoraron significativamente en velocidad del procesamiento, capacidad de aprendizaje y memoria visuoespacial y control inhibitorio. Además, es importante destacar que este grupo de pacientes mostró también una mejoría significativa en el estado de ánimo.

Trabajos anteriores han descrito que tanto los ejercicios físicos $[18,19,26,27]$ como los programas de rehabilitación cognitiva [28-30] resultan beneficiosos en la optimización de las funciones cognitivas en pacientes con EM. No obstante, se ha observado una limitada evidencia sobre la eficacia en las funciones cognitivas del uso de tratamientos combinados (físico y cognitivo). En este sentido, los resultados obtenidos refuerzan la idea de que el tratamiento combinado es eficaz para mejorar el rendimiento cognitivo en pacientes con EM.

Los resultados de mejoría en el control inhibitorio en el GE mediante la prueba Stroop interferen- cia palabra-color coinciden con los descritos por Valencia et al [31], quienes probaron la eficacia de un entrenamiento combinado (entrenamiento de memoria y psicomotricidad) en adultos mayores sanos.

Del mismo modo, una revisión sistemática sintetizó los resultados de los entrenamientos combinados mediante ejercicios físicos y cognitivos, en este caso en personas de edad avanzada con diagnóstico de deterioro cognitivo leve y demencia [32]. Se incluyeron ocho trabajos, en los cuales la rehabilitación consistía en enseñar estrategias educativas, desarrollar ejercicios físicos diversos y realizar tareas cognitivas. Los resultados muestran que la combinación de los ejercicios físicos y la intervención cognitiva contribuye significativamente a la optimización del funcionamiento cognitivo en general, incluyendo la atención, la memoria, las funciones ejecutivas y los estados funcionales de los pacientes con deterioro cognitivo leve y demencia [32-34].

Un estudio reciente ha mostrado la asociación entre alteraciones en la memoria visuoespacial con 
las restricciones en la participación en los pacientes con EM, específicamente en el desempeño en el área laboral [35]. Brissart et al [28] sugirieron la importancia de estimular los procesos visuoespaciales para obtener beneficios sobre otras funciones cognitivas en los pacientes con EM. No obstante, pocos estudios han utilizado tratamientos combinados para mejorar esta función. En otras poblaciones clínicas se ha evidenciado que las habilidades visuoespaciales pueden entrenarse en el ámbito de la rehabilitación cognitiva mediante tareas duales cognitivomotoras con estímulo visuoespacial [36]. Estos resultados coinciden con nuestra investigación, donde se utilizó la modalidad visuomotora (tareas del TaDiCS) para estimular las habilidades de adquisición de la información visuoespacial en los pacientes del GE.

Diversos estudios han comprobado el efecto positivo de los ejercicios físicos cuando se emplean antes de la intervención cognitiva [32,34]. Algunos autores plantean que es razonable que los ejercicios físicos se realicen antes de las sesiones de intervención cognitiva, ya que, al parecer, el ejercicio físico prepara al cerebro y sus procesos compensatorios, y de este modo las sesiones de entrenamiento cognitivo resultan ser más eficaces [32]. Nuestro estudio consideró los anteriores planteamientos y las sesiones de entrenamiento cognitivo se realizaron después de los ejercicios aeróbicos, con un intervalo de 30 minutos que permitiera la recuperación de los pacientes. Así, los ejercicios físicos pueden considerarse como un complemento útil para facilitar el efecto del entrenamiento cognitivo.

Debido a que la depresión suele ser un síntoma común en la EM y frecuentemente aparece asociada al deterioro cognitivo [37], es importante destacar la mejora de la sintomatología depresiva en pacientes que realizan tanto ejercicios físicos como cognitivos $[18,29,30]$. Estos resultados son consistentes con los de nuestro estudio, donde hemos observado una mejoría significativa del estado de ánimo de ambos grupos de pacientes (GE y GC).

Una limitación de la presente investigación es la no inclusión de un grupo control puro (sin ningún tipo de tratamiento); por tanto, aunque los resultados son consistentes, no es posible concluir de forma certera la validez de la terapia combinada. Futuros trabajos se deben considerar con muestras más grandes de pacientes y otros diseños experimentales, que incorporen diferentes modalidades de tratamiento (cognitivo, físico y la combinación de ambos), así como grupos de pacientes sin tratamiento.

En conclusión, los resultados del presente estudio piloto indican que el programa de entrenamien- to combinado (físico y cognitivo) resulta más eficaz que el entrenamiento físico en la optimización de las funciones cognitivas. Estos datos conducen a pensar que en los pacientes con EM es mejor realizar programas de entrenamientos combinados donde se trabajen todas las funciones alteradas, tanto físicas como cognitivas, ya que la mejora física parece repercutir en la mejora cognitiva y viceversa. La evidencia preliminar apoya que la interacción de ejercicios físicos y tareas de rehabilitación cognitiva suele ser una relación potencial que garantiza a la rehabilitación mayor alcance para mejorar el estado funcional durante el curso clínico de la EM [38].

\section{Bibliografía}

1. Chiaravalloti ND, DeLuca J. Cognitive impairment in multiple sclerosis. Lancet Neurol 2008; 7: 1139-51.

2. Rocca MA, Amato MP, De Stefano N, Enzinger CH, Geurts JJ, Penner I, et al. Clinical and imaging assessment of cognitive dysfunction in multiple sclerosis. Lancet Neurol 2015; 14: 302-17.

3. Kalmar JH, Gaudino EA, Moore NB, Halper J, Deluca J. The relationship between cognitive deficits and everyday functional activities in multiple sclerosis. Neuropsychology 2008; 22: 442-9.

4. Rao SM, Leo GI, Ellington L, Nauertz T, Bernardin L, Unverzagt F. Cognitive dysfunction in multiple sclerosis II. Impact on employment and social functioning. Neurology 1991; 41: 692-6.

5. Bergendal G, Fredrikson S, Almkvist O. Selective decline in information processing in subgroups of multiple sclerosis: an 8-year longitudinal study. Eur Neurol 2007; 57: 193-202.

6. Macías-Delgado Y, Jiménez-Morales RM, Fundora R, Gómez AO, Sebrango CR, Baldomir RR. Fatiga cognitiva en la esclerosis múltiple. Relación con la fatiga subjetiva y memoria de trabajo. Revista Cubana de Medicina Física y Rehabilitación 2012; 4: 95-104.

7. Benedict RH, Cookfair D, Gavett R, Gunther M, Munschauer F, Garg N, et al. Validity of the minimal assessment of cognitive function in multiple sclerosis (MACFIMS). J Int Neuropsychol Soc 2006; 12: 549-58.

8. Chiaravalloti ND, DeLuca J. The influence of cognitive dysfunction on benefit from learning and memory rehabilitation in MS: a sub-analysis of the MEMREHAB trial. Mult Scler 2015; 21: 1575-82.

9. Goverover Y, Basso M, Wood H, Chiaravalloti ND, DeLuca J. Examining the benefits of combining two learning strategies on recall of functional information in persons with multiple sclerosis. Mult Scler 2011; 17:1488-97.

10. Plohmann AM, Kappos L, Ammann W, Thordai A, Wittwer A, Huber S, et al. Computer assisted retraining of attentional impairments in patients with multiple sclerosis. J Neurol Neurosurg Psychiatry 1998; 64: 455-62.

11. Amato MP, Goretti B, Viterbo RG, Portaccio E, Niccolai C, Hakiki B, et al. Computer-assisted rehabilitation of attention in patients with multiple sclerosis: results of a randomized, double-blind trial. Mult Scler 2014; 20: 91-8.

12. Fink F, Rischkau E, Butt M, Klein J, Eling P, Hildebrandt $H$. Efficacy of an executive function intervention programme in MS: a placebo-controlled and pseudo-randomized trial. Mult Scler 2010; 16: 1148-51.

13. O'Brien AR, Chiaravalloti N, Goverover Y, Deluca J. Evidencedbased cognitive rehabilitation for persons with multiple sclerosis: a review of the literature. Arch Phys Med Rehabil 2008; 89: 761-9.

14. Mitoloa M, Venneri A, Wilkinson ID, Sharrack B. Cognitive rehabilitation in multiple sclerosis: a systematic review. J Neurol Sci 2015; 354: 1-9.

15. Small GW, Silverman DH, Siddarth P, Ercoli LM, Miller KJ, Lavretsky H, et al. Effects of a 14-day healthy longevity lifestyle 
program on cognition and brain function. Am J Geriatr Psychiatry 2006; 14: 538-45.

16. Jedrziewski MK, Ewbank DC, Wang H, Trojanowski JQ. The impact of exercise, cognitive activities, and socialization on cognitive function: results from the National Long-Term Care Survey. Am J Alzheimers Dis Other Demen 2014; 29: 372-8.

17. Beier M, Bombardier C, Hartoonian N, Motl R, Kraft G. Improved physical fitness correlates with improved cognition in MS. Arch Phys Med Rehabil 2014; 95: 1328-34.

18. Briken S, Gold SM, Patra S, Vettorazzi E, Harbs D, Tallner A et al. Effects of exercise on fitness and cognition in progressive MS: a randomized, controlled pilot trial. Mult Scler 2014; 20: 382-90.

19. Motl R, Gappmeier E, Nelson K, Benedict R. Physical activity and cognitive function in MS. J Sport Exerc Psychol 2011; 33 734-41.

20. McDonald WI, Compston A, Edan G, Goodkin D, Hartung HP, Lublin FD, et al. Recommended diagnostic criteria for multiple sclerosis: guidelines from the International Panel on the Diagnosis of MS. Ann Neurol 2001; 50: 121-7.

21. Sepulcre J, Vanotti S, Hernández R, Sandoval G, Cáceres F, Garcea $\mathrm{O}$, et al. cognitive impairment in patients with multiple sclerosis using the Brief Repeatable Battery-Neuropsychology test. Mult Scler 2006; 12: 187-95.

22. Rao SM, Cognitive Function Study Group. A manual for the Brief Repeatable Battery of Neuropsychological Test in multiple sclerosis. New York: National Multiple Sclerosis Society; 1990.

23. Goldman Consensus Group. The Goldman Consensus statement on depression in multiple sclerosis. Mult Scler 2005; 11: 328-37.

24. Sohlberg MM, Mateer CA. Effectiveness of an attention training program. J Clin Exp Neuropsychol 1987; 9: 117-30.

25. Luria AR. El cerebro y acción. La Habana: Pueblo y Educación; 1989.

26. Sandroff BM, Klaren RE, Pilutti LA, Dlugonski D, Benedict RHB, Motl RW. Randomized controlled trial of physical activity, cognition and walking in multiple sclerosis. J Neurol 2014; 261: 363-72

27. Sandroff BM. Exercise and cognition in multiple sclerosis: the importance of acute exercise for developing better interventions, Neurosci Biobehav Rev 2015; 59: 173-83.

28. Brissart H, Leroy M, Morele E, Baumann C, Spitz E,
Debouverie M. Cognitive rehabilitation in multiple sclerosis. Neurocase 2013; 19: 553-65.

29. Gich J, Freixanet J, García R, Vilanova JC, Genís D, Silva Y, et al. A randomized, controlled, single-blind, 6-month pilot study to evaluate the efficacy of MS-Line!: a cognitive rehabilitation programme for patients with multiple sclerosis. Mult Scler 2015; 21: 1332-43.

30. Mattioli F, Stampatori C, Zanotti D, Parrinello G, Capra R. Efficacy and specificity of intensive cognitive rehabilitation of attention and executive functions in multiple sclerosis. J Neurol Sci 2010; 288: 101-5.

31. Valencia C, López-Alzate E, Tirado V, Zea-Herrera MD, Lopera F, Rupprecht R, et al. Efecto de un entrenamiento combinado de memoria y psicomotricidad en adultos mayores. Rev Neurol 2008; 46: 465-71.

32. Law LL, Barnett F, Yau MK, Gray MA. Effects of combined cognitive and exercise interventions on cognition in older adults with and without cognitive impairment: a systematic review. Ageing Res Rev 2014; 15: 61-75.

33. Fabre C, Chamari K, Mucci P, Massé-Biron J, Préfaut C. Improvement of cognitive function by mental and/or individualized aerobic training in healthy elderly subjects. Int J Sports Med 2002; 23: 415-21.

34. Oswald WD, Gunzelmann T, Rupprecht R, Hagen B. Differential effects of single versus combined cognitive and physical training with older adults: the SimA study in a 5-year perspective. Eur J Ageing 2006; 3: 179-92.

35. Goverover Y, Strober L, Chiaravalloti N, DeLuca J. Factors that moderate activity limitation and participation restriction in people with multiple sclerosis. Am J Occup Ther 2015; 69: 6902260020p1-9.

36. Kounti F, Bakoglidou E, Agogiatou C, Emerson NB, Lazarus L, Tsolaki M. RHEA: a nonpharmacological cognitive training intervention in patients with mild cognitive impairment: a pilot study. Top Geriatr Rehabil 2011; 27: 289-300.

37. Arnett PA, Barwick FH, Beeney JE. Depression in multiple sclerosis: review and theoretical proposal. J Int Neuropsychol Soc 2008; 14: 691-724.

38. Motl RW, Sandroff BM, DeLuca J. Exercise training and cognitive rehabilitation: a symbiotic approach for rehabilitating walking and cognitive functions in multiple sclerosis? Neurorehabil Neural Repair 2016; 30: 499-511.

\section{Cognitive training combined with aerobic exercises in multiple sclerosis patients: a pilot study}

Introduction. The scientific evidences associated to the effectiveness of different techniques of cognitive rehabilitation are still contradictory.

Aim. To compare a program of combined training (physical and cognitive) in front of a program of physical training and to observe their effectiveness about the optimization of the cognitive functions in patients with multiple sclerosis (MS).

Patients and methods. It was carried out an experimental study in 32 patients with MS. The patients were distributed in two groups: 16 to the experimental group (combined cognitive training with aerobic exercises) and 16 patients to the control group (aerobic exercises). The intervention was planned for six weeks combining cognitive tasks by means of a game of dynamic board of cubes and signs $\left(\operatorname{TaDiCS}{ }^{\circledR}\right.$ ) and a program of aerobic exercises. The Brief Repeatable Battery of Neuropsychological Test and the Stroop Test were applied to evaluate the cognitive yield. Also, the Beck Depression Inventory was administered.

Results. There were found significant differences in the intergrupal analysis after the intervention in the variable learning and visuoespacial long term memory $(p=0.000)$, attention $(p=0.026)$ and inhibitory control $(p=0.007)$. Also, in the intragroup analysis there were found significant differences in these variables and information processing speed in the group that received the combined training. These patients also showed a significant improvement in the emotional state $(p=0.043)$.

Conclusion. The cognitive training combined with the aerobic exercises is effective to improve the cognitive performance.

Key words. Aerobic exercises. Attention. Cognitive function. Cognitive training. Information processing speed. Learning and memory. Multiple sclerosis. 